Trauma Berufskrankh 2008 · 10[Suppl 1]:9-12 DOI 10.1007/s10039-007-1220-9

Online publiziert: 30. März 2007

๑) Springer Medizin Verlag 2007

\title{
T. Kittner
}

Klinik für Radiologie, Krankenhaus Dresden-Friedrichstadt, Städtisches Klinikum, Akademisches Lehrkrankenhaus der TU Dresden

\section{Spezielle radiologische Diagnostik der verletzten Schulter}

\section{Spezielle radiologische Diagnostik}

Die Diagnostik der verletzten Schulter hat in den letzten Jahren mit der ständigen Weiterentwicklung der radiologischen Technik viele neue Aspekte erfahren.

Das konventionelle Röntgen unterlief einer Digitalisierung beginnend mit der Einführung der Speicherfoliensysteme bis hin zur Entwicklung der Festkörperdetektortechnik. Durch die damit verbundenen Nachbearbeitungseigenschaften kam es $\mathrm{zu}$ einer weitestgehenden Entkoppelung der Bildqualität von der Belichtung, was Wiederholungsaufnahmen kaum noch erforderlich macht. Die Bildverteilung über schnelle Datennetze generiert filmlose Krankenhäuser und ermöglicht eine schnelle Verfügbarkeit der Aufnahmen für alle an der Behandlung Beteiligten.

Mit der Einführung der Mehrzeilenspiralcomputertomographie (MS-CT) in die klinische Praxis wurde die CT wahrhaft dreidimensional, da bei dieser Modifikation die räumliche Auflösung in allen 3 Ebenen gleich hoch ist. Dies ist Voraussetzung für sekundäre, multiplanare Rekonstruktionen mit einer beeindruckenden Brillanz (• Abb. 1).

Die Magnetresonanztomographie (MRT) steigerte infolge neuer Hard- und Software ihr räumliches Auflösungsvermögen bis in den Submillimeterbereich. Die Gelenkdiagnostik, d. h. die Darstellung von ossären, kartilaginären und ligamentären Strukturen, wurde hiermit erheblich verbessert, wodurch die Methode als Goldstandard der muskuloskelettalen Bildgebung eine weitere Erhöhung der Treffsicherheit erfuhr.
Verschiedenste Verfahren stehen zur Diagnostik der verletzten Schulter zur Verfügung:

- Sonographie

- Tomographie

- Arthrographie (Röntgen-/CT-/MRArthrographie)

- Projektionsradiographie

- Computertomographie

- Magnetresonanztomographie

\section{Sonographie}

Der Ultraschall ist aufgrund seiner breiten Verfügbarkeit und geringen Kosten Methode der ersten Wahl bei:

- der Abklärung von Erkrankungen der Sehnen (z. B. Supraspinatussehne) bzw. der Bänder oder - der Detektion von Gelenkergüssen.

Neue Modifikationen wie das Tissue-Harmonic-Imaging erlauben eine detailgetreue Abgrenzbarkeit der labralen Strukturen, wobei unverändert das Problem der Schallreflexion durch Luft oder Knochen bestehen bleibt, was eine grundlegende Limitation des Verfahrens beinhaltet.

\section{Tomographie}

Die konventionelle Schichtuntersuchung wurde mehr und mehr durch die breite Einführung der Computertomographie verdrängt. Aufgrund ihrer gegenüber der CT begrenzten Aussagekraft wird sie heute nur noch in Ausnahmefällen eingesetzt. Die fehlende Möglichkeit der sekundären, multiplanaren Rekonstruktion und die Fixierung auf nur eine Ebene pro Schichtung haben sehr schnell zu fehlenden Nachfragen nach der zeitaufwändigen Methode geführt.

\section{Konventionelles Röntgen (Projektionsradiographie)}

Die Röntgenuntersuchung der verletzten Schulter in 2 Ebenen ist auch heute noch die initiale Standarddiagnostik zur Abklärung von:

- akuten Erkrankungen wie Frakturen und Luxationen oder

- chronischen Erkrankungen im Rahmen von Instabilitäten (Subluxationen) oder des subakromialen Schmerzsyndroms (z. B. Impingement).

Im Akut-Trauma-Stadium wird als 2. Ebene zumeist mechanisch- oder schmerzbedingt nur die mit Überlagerungen verbundene Skapula-Tangential-Y-Aufnahme möglich sein, die sonst durch ein im rein axialen Strahlengang angefertigtes Bild ersetzt wird. Für die bessere Beurteilung des Glenoids hinsichtlich des Vorliegens einer knöchernen Bankart-Läsion empfiehlt sich die Pfannenprofilaufnahme nach Bernageau ( $\bullet$ Abb. 2), für die Einschätzung einer Einengung des subakromialen Raums und Supraspinatussehnenauslasses die modifizierte Y-Aufnahme nach Neer/Morrison ( $\bullet$ Abb. 3).

Die konventionelle Arthrographie ohne Post-Arthro-CT hat innerhalb der ra- 


\section{Die verletzte Schulter}

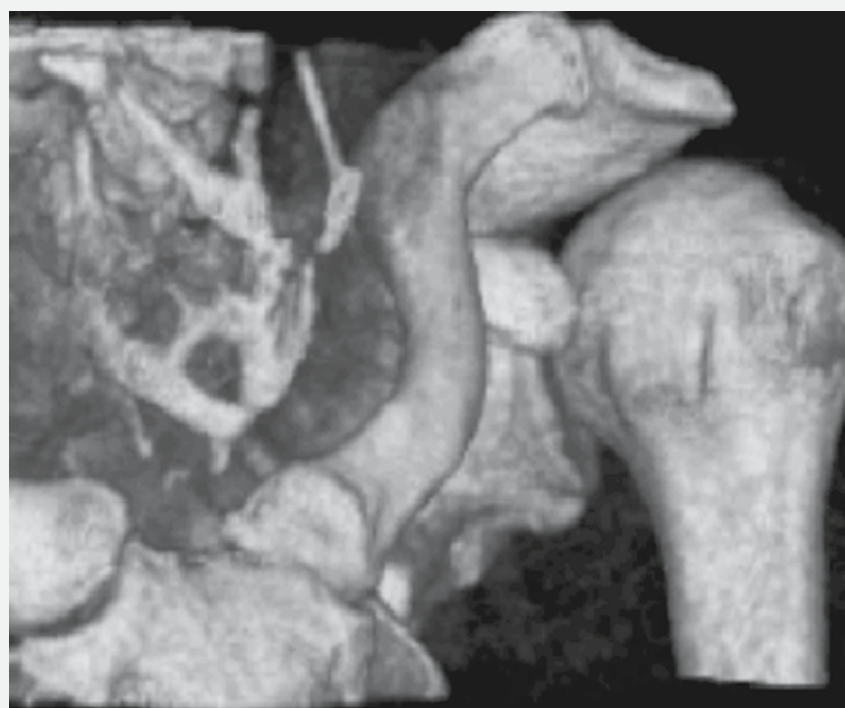

Abb. 1 \ 16-Zeilen-Spiral-CT mit Volume-Rendering-Rekonstruktion der Schulter

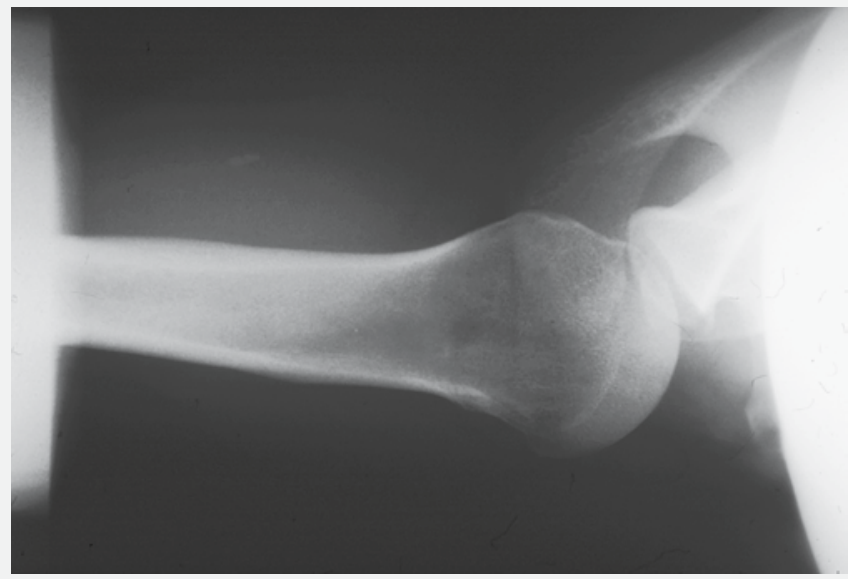

Abb. $2 \Delta$ Pfannenprofil nach Bernageau

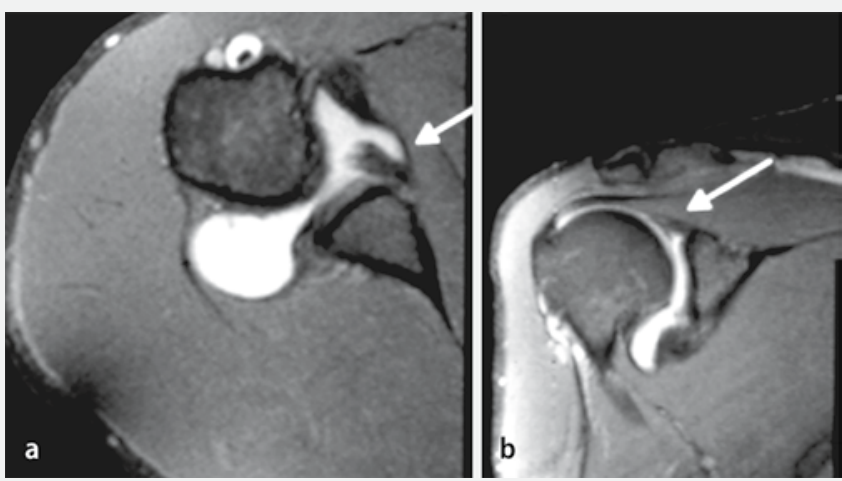

Abb. 5 \ Perthes-Läsion (a), SLAP-Läsion (Grad II nach Snyder) (b)

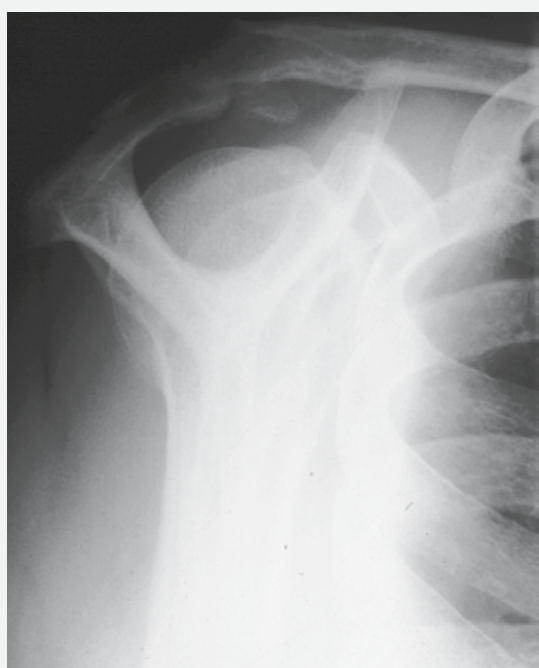

Abb. $3 \Delta$ Skapula-Y-Aufnahme (Neer/Morisson); subakromiales Ossikel
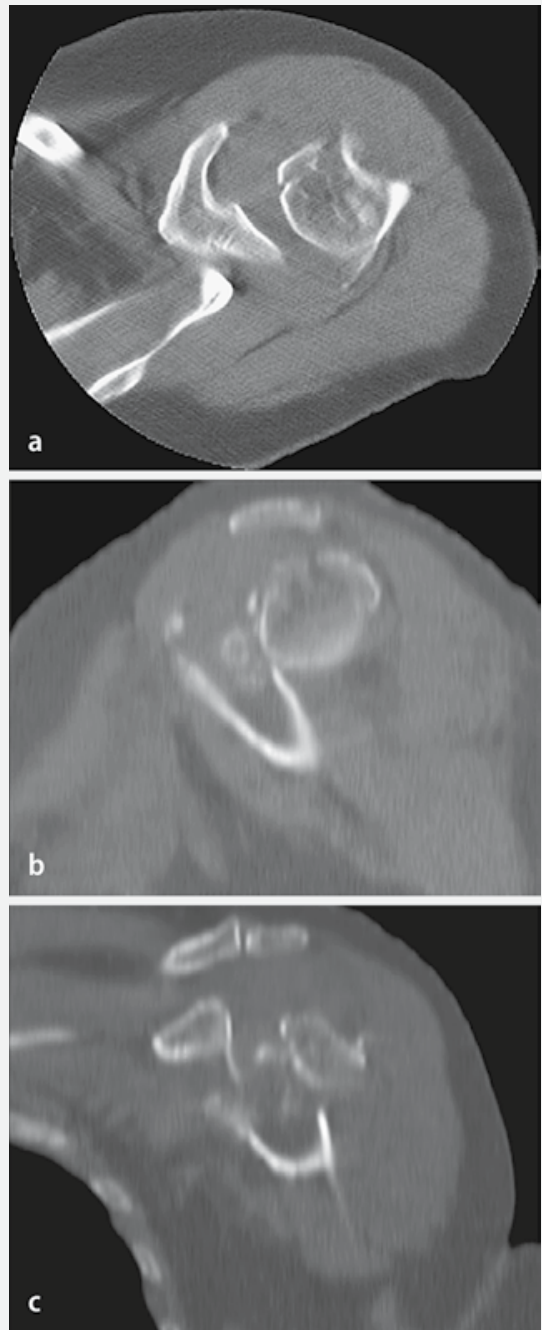

Abb. $4 \Delta$ Axiale primäre CT-Schicht (a), sagittale (b) und koronare Rekonstruktion (c) 
diologischen Palette ihre Bedeutung verloren, da komplette Rotatorenmanschettenrupturen nichtinvasiv mit Ultraschall oder MRT eleganter diagnostizierbar sind.

\section{Computertomographie}

Sie besitzt gegenüber der Projektionsradiographie den Vorteil, das zu untersuchende Objekt überlagerungsfrei abbilden zu können (• Abb.4a). Diese Eigenschaft, gepaart mit der Möglichkeit von multiplanaren Rekonstruktionen in jeder beliebigen Ebene des Raums ( $(\bullet$ Abb. 4b, c), macht sie zur Methode der Wahl bei komplizierten Gelenkverletzungen. Hiermit können dislozierte Fragmente, Trümmerzonen oder Impressionen besser visualisiert werden. Dies gilt insbesondere bei der initial nicht selten fehldiagnostizierten dorsalen Luxation, zum einen um die Diagnose zu bestätigen, zum anderen bei der Darstellung der reversen Hill-Sachs- und Bankart-Läsionen im präoperativen Untersuchungsgang.

Postoperativ gelingt es heute durch neue, artefaktsupprimierende Rekonstruktionsalgorithmen auch bei liegendem Osteosynthesematerial die Lage der Implantate zuverlässig zu bestimmen [1].

\section{Magnetresonanztomographie}

Aufgrund ihrer Nichtinvasivität, des hohen Weichteilkontrasts und der primär multiplanaren Abbildungsmöglichkeit hat sich die MRT auf dem Gebiet der muskuloskelettalen Schnittbilddiagnostik den ersten Platz unter den bildgebenden Verfahren erobert, wenn es um die Beurteilung von kapsulo-labralen Strukturen, Bändern und Knorpel geht.

Insbesondere bei Instabilitäten (Subluxationen) muss diese Nichtinvasivität jedoch zugunsten der direkten MR-Arthrographie mit der intraartikulären Instillation eines Gadolinium-Kochsalz-Gemisches aufgegeben werden. Hierdurch wird ein aus der Arthroskopie bekannter Effekt der Distension des Gelenkes erreicht, womit insbesondere kleinere Läsionen am antero-inferioren Kapsel-Labrum-Komplex (Bankart) oder am superioren Labrum (SLAP) infolge des Eintritts des applizierten Gemisches in die entstandenen

Trauma Berufskrankh 2008 · 10[Suppl 1]:9-12 DOI 10.1007/s10039-007-1220-9

(c) Springer Medizin Verlag 2007

T. Kittner

Spezielle radiologische Diagnostik der verletzten Schulter

\section{Zusammenfassung}

In Abhängigkeit von der Art und der Ursache der Schulterverletzung empfehlen sich unterschiedliche Diagnosemethoden. Bei rezidivierender Luxation sollten ebenso wie bei multidirektionaler, bilateraler Instabilität Röntgenaufnahmen in 2 Ebenen angefertigt werden, ggf. sind Spezialprojektionen (Pfannenprofil nach Bernageau, Skapula-Y-Aufnahme nach Neer/Morisson) erforderlich. Bei (Mehrfragment)-Frakturen sowie bei fixierter dorsaler Luxation sollten die Röntgenaufnahmen in 2 Ebenen durch eine Computertomographie ergänzt werden. Besteht der Verdacht auf ei-

ne Subluxation, ist die MR-Arthrographie die Diagnosemethode der Wahl. Das subakromiale Schmerzsyndrom inklusive Rotatorenmanschettenpathologie wird am besten mit Hilfe von Röntgenaufnahmen in 2 Ebenen, ggf. mit Spezialprojektionen (Skapula-Y-Aufnahme nach Neer/Morisson), sowie mittels Sonographie und ggf. MRT abgeklärt.

\section{Schlüsselwörter}

Schulterverletzung $\cdot$ Röntgen .

Computertomographie - MR-Arthrographie Sonographie

\section{Specific imaging techniques for diagnosis of different shoulder injuries}

\section{Abstract}

The most appropriate method of diagnosis varies depending on the kind and the aetiology of shoulder injury. In the case of recurring dislocation, as for multidirectional bilateral instability, $\mathrm{X}$-ray exposures in two planes should be performed; it may be that special projections (acetabular profile after Bernageau; Y view of scapula after Neer/Morrison) are needed. In the case of (multiply) fragmented fractures and in that of fixed dorsal dislocation the radiographs in two planes should be supplemented by computed tomography. When subluxation is suspected
MR arthrography is the method of choice for diagnosis. Subacromial pain syndrome including rotator cuff pathology is best investigated with the aid of X-rays in two planes, if necessary with specific projections ( $Y$ view of the scapula as per Neer/Morrison), ultrasonography, and if appropriate, MRT.

\section{Keywords}

Shoulder injury · Radiography · Computed tomography · MR arthrography .

Ultrasonography 
Taschen erheblich besser dargestellt werden können, als dies mit der nichtarthrographischen MRT möglich wäre [3].

Partiell verheilte Läsionen (Perthes) bedürfen einer Untersuchung unter mechanischen Stressbedingungen, indem der Patient, die Handfläche unter dem Hinterkopf liegend, in einer Abduktionsund Außenrotationsposition (ABER: „abduction and external rotation") untersucht wird. Nur so können diese mechanisch instabilen Veränderungen MR-tomographisch erkannt werden (• Abb. 5).

\section{Fazit}

In Abhängigkeit vom Bild und der Ursache der Schulterverletzung empfehlen sich unterschiedliche Methoden für die spezielle radiologische Diagnostik [2]:

\section{Rezidivierende Luxation}

- Röntgenaufnahme in 2 Ebenen

- Ggf. Spezialprojektionen (Pfannenprofil nach Bernageau; Skapula-YAufnahme nach Neer/Morisson)

2. Fraktur (Mehrfragment)

- Röntgenaufnahme in 2 Ebenen

- CT

3. Fixierte dorsale Luxation

- Röntgenaufnahme in 2 Ebenen

- CT

4. Subluxationen

- MR-Arthrographie

5. Multidirektionale, bilaterale Instabilität

- Röntgenaufnahme in 2 Ebenen

- Ggf. Spezialprojektionen (Pfannenprofil nach Bernageau; Skapula-Y-

Aufnahme nach Neer/Morisson)

- CT/MRT ohne Benefit

6. Subakromiales Schmerzsyndrom inklusive Rotatorenmanschettenpathologie

- Röntgenaufnahme in 2 Ebenen

- Ggf. Spezialprojektionen (SkapulaY-Aufnahme nach Neer/Morisson)

- Sonographie

- Ggf. MRT

\section{Korrespondierender Autor}

\section{PD Dr. T. Kittner}

Klinik für Radiologie,

Krankenhaus Dresden-Friedrichstadt, Städtisches Klinikum,

Akademisches Lehrkrankenhaus der TU Dresden Friedrichstraße 41, 01067 Dresden

kittner-th@khdf.de
Interessenkonflikt. Es besteht kein Interessenkonflikt. Der korrespondierende Autor versichert, dass keine Verbindungen mit einer Firma, deren Produkt in dem Artikel genannt ist, oder einer Firma, die ein Konkurrenzprodukt vertreibt, bestehen. Die Präsentation des Themas ist unabhängig und die Darstellung der Inhalte produktneutral.

\section{Literatur}

1. Burkhardt M, Gänsslen A, Uder M et al. (2003) Neue Möglichkeiten der Visualisierung von Frakturen mittels CT: Rekonstruktionen, 3D-Planungen. Schwierige Gelenkverletzungen - Modernes Management - Verbesserte Bildgebung und Operationsplanung bei Gelenkverletzungen. Zentralbl Chir 128: 34-39

2. Kittner T, Hartmann A (2000) Schulterdiagnostik. RöFo Fortschr Geb Röntgenstr Neuen Bildgeb Verfahr 170: 214

3. Kittner T, Bottesi M, Abolmaali N et al. (1999) MR arthrography imaging in patients with suspected anterior glenohumeral instability: anatomic and arthroscopic correlation. Radiology 213: 539 